\title{
High Risk Chronic Myelomonocytic Leukemia
}

National Cancer Institute

\section{Source}

National Cancer Institute. High Risk Chronic Myelomonocytic Leukemia. NCI Thesaurus.

Code C156293.

Chronic myelomonocytic leukemia with cytogenetic abnormalities including trisomy 8 , complex karyotypes, monosomy 7, and del17q. Mutations in RUNX1, NRAS, SETBP1, and ASXL1 are independently associated with inferior overall survival time. (Oncology Letters $15: 7132-7138,2018)$ 